\title{
Inhibition of HSP90 reverses STAT3-mediated muscle wasting in cancer cachexia mice.
}

Mengyuan $\mathrm{Niu}^{1}$, Shiyu Song ${ }^{1}$, Zhonglan $\mathrm{Su}^{2}$, Lulu $\mathrm{Wei}^{1}$, $\mathrm{Li} \mathrm{Li}^{1}$, Wenyuan $\mathrm{Pu}^{1}$, Chen

Zhao $^{1}$, Yibing Ding ${ }^{1}$, Jinglin Wang ${ }^{3}$, Wangsen $\mathrm{Cao}^{1}$, Qian $\mathrm{Gao}^{1}$, and hongwei Wang ${ }^{1}$

${ }^{1}$ Nanjing University

${ }^{2}$ Jiangsu Province People's Hospital and Nanjing Medical University First Affiliated

Hospital

${ }^{3}$ Nanjing University Medical School Affiliated Nanjing Drum Tower Hospital

April 10, 2021

\begin{abstract}
Cancer cachexia is one of the most common causes of death among cancer patients, no effective anti-cachectic treatment is currently available. In experimental cachectic animal models, aberrant activation of STAT3 in skeletal muscle has been found to contribute to muscle wasting. However, its clinical association, the factors regulating STAT3 activation, and the molecular mechanisms remain incompletely understood. Here, we show that an enhanced interaction between activated STAT3 and HSP90, which were observed in the skeletal muscle of cancer cachexia patients, is a crucial event for the development of cachectic muscle wasting. Administration of HSP90 inhibitors 17DMAG and PU-H71 alleviated the muscle wasting in C26 tumor-bearing cachectic mice or the myotube atrophy of $\mathrm{C} 2 \mathrm{C} 12$ cells induced by $\mathrm{C} 26$ conditional medium. A mechanistic study indicated that in cachectic skeletal muscle, prolonged STAT3 activation transactivated FOXO1 by binding directly to its promoter and triggered the muscle wasting in a FOXO1-dependent manner; Our results demonstrate that the HSP90/STAT3/FOXO1 axis plays a critical role in the cachectic muscle wasting, which might serve as potential therapeutic target for the treatment of cancer cachexia.
\end{abstract}

\section{Inhibition of HSP90 reverses STAT3-mediated muscle wasting in cancer cachexia mice}

Mengyuan Niu ${ }^{1 \#}$, Shiyu Song ${ }^{1 \#}$, Zhonglan $\mathrm{Su}^{2}$, Lulu Wei ${ }^{1}$, $\mathrm{Li} \mathrm{Li}^{1}$, Wenyuan $\mathrm{Pu}^{1}$, Chen Zhao ${ }^{1}$, Yibing Ding $^{1}$, Jinglin Wang ${ }^{1}$, Wangsen $\mathrm{Cao}^{1}$, Qian $\mathrm{Gao}^{1 *}$, Hongwei Wang ${ }^{1 *}$

1.Center for Translational Medicine and Jiangsu Key Laboratory of Molecular Medicine, Medical School of Nanjing University, Nanjing 210093, P.R. China

2.Department of Dermatology, the First Affiliated Hospital of Nanjing Medical University, Nanjing 210029, P.R. China

\# Equal contribution

* Address for Correspondence:

Hongwei Wang

Center for Translational Medicine and Jiangsu Key Laboratory of Molecular Medicine, Medical School of Nanjing University,

Nanjing 210093, PR China

E-mail: hwang@nju.edu.cn; 
Tel: +862583594755 ; Fax: +86 2583594755

\section{Availability of data}

The data that support the findings of this study are available from the corresponding author upon reasonable request. Some data may not be made available because of privacy or ethical restrictions.

\section{Abstract \\ Background and purpose:}

Cancer cachexia is one of the most common causes of death among cancer patients, no effective anti-cachectic treatment is currently available. In experimental cachectic animal models, aberrant activation of STAT3 in skeletal muscle has been found to contribute to muscle wasting. However, its clinical association, the factors regulating STAT3 activation, and the molecular mechanisms remain incompletely understood.

\section{Experimental approach:}

The expression of HSP90 and the activation of STAT3 were detected in muscle from the patients with cancer cachexia or the tumour-bearing cachectic mice. HSP90 inhibitors, including 17DMAG and PU-H71, were administered to cachexic mice, and cachexia parameters, such as weight loss, food intake, survival rate, body mass composition, serum metabolites, muscle wasting pathology and catabolic activation, were measured and analysed. The in vitro coculture of $\mathrm{C} 2 \mathrm{C} 12$ myotube cells with $\mathrm{C} 26$ conditioned media (CM) was performed to address the pathological mechanism of catabolic muscle wasting. The roles of HSP90, STAT3 and FOXO1 in myotube atrophy were explored via overexpression or knockdown.

\section{Results:}

Here, we show that an enhanced interaction between activated STAT3 and HSP90, which were observed in the skeletal muscle of cancer cachexia patients, is a crucial event for the development of cachectic muscle wasting. Administration of HSP90 inhibitors 17DMAG and PU-H71 alleviated the muscle wasting in C26 and LLC tumor-bearing cachectic mice models or the myotube atrophy of $\mathrm{C} 2 \mathrm{C} 12$ cells induced by $\mathrm{C} 26$ conditional medium. Prolonged STAT3 activation transactivated FOXO1 by binding directly to its promoter and triggered the muscle wasting in a FOXO1-dependent manner in muscle cells.

\section{Conclusion and implications:}

Our results demonstrate that the HSP90/STAT3/FOXO1 axis plays a critical role in the cachectic muscle wasting, which might serve as potential therapeutic target for the treatment of cancer cachexia.

\section{Bullet point summary}

\section{What is already known}

Increased STAT3 activation (in muscle) in multiple types of experimental cancer cachexia; Enhanced FOXO1 activation in skeletal muscle triggered the muscle atrophy by activation of downstream target genes, including Atrogin-1 and MuRF1.

\section{What this study adds}

HSP90 interacts with STAT3 and promotes its persistent activation in cachectic muscles. Knocking down of HSP90 and its inhibitor disrupts the interaction and attenuates muscle atrophy through the downregulation of STAT3/FOXO1 axis.

\section{Clinical significance}

Blocking HSP90 activity could potentially prevent or reverse cancer cachexia development.

\section{Keywords}

Cancer cachexia; Muscle wasting; HSP90; STAT3; FOXO1; 


\section{Introduction}

Cancer cachexia is a devastating metabolic syndrome that affects up to $80 \%$ of cancer patients and accounts for nearly $20 \%$ of all cancer-related deaths. Skeletal muscle wasting is one of the most crucial pathological events in cancer cachexia. As a highly plastic tissue, skeletal muscle can change its mass, function, and metabolism in response to various endogenous or exogenous stimuli. Current evidence indicates that the imbalance between catabolic and anabolic responses, the disorder of protein synthesis and degradation pathways, including both the ubiquitin-proteasome system (UPS) and the autophagy-lysosome pathway (ALP), are the major causes of cachectic muscle wasting (Bilodeau, Coyne \& Wing, 2016). In cancer cachexia, UPS activation is thought to mediate muscle atrophy, and the enhanced expression of musclespecific E3 ubiquitin ligases, such as muscle-atrophy-F-box (MAFbx/Atrogin-1) and muscle-RING-finger-1 (MuRF1), are hallmarks of this process (Bodine et al., 2001; Guadagnin, Mazala \& Chen, 2018; Guo, Wang, Wang, Qiao \& Tang, 2017). However, the upstream activators of the protein degradation pathway and the molecular mechanisms involved in muscle wasting in cancer cachexia are still largely unknown.

Signal transducer and activator of transcription 3 (STAT3) plays a critical role in cancer cachexia, and increased STAT3 activation (in muscle) has been found in multiple types of experimental cancer cachexia. (Ma, Sanchez, Hall, Tremblay, Di Marco \& Gallouzi, 2017; Mubaid et al., 2019; Silva et al., 2015) In experimental cancer cachexia, muscle-specific STAT3 depletion or JAK2/STAT3 pathway inhibition can reverse the skeletal muscle wasting phenotype(Bonetto et al., 2012). However, its clinical association, the reason for prolonged STAT3 activation in cachectic muscles, and its contribution to pathological anabolic responses in muscle still need to be defined.

HSP90 (heat shock protein 90) is an evolutionarily conserved molecular chaperone that is essential for cell growth, proliferation, transformation, proliferation, and survival under normal and stress conditions (Schopf, Biebl \& Buchner, 2017). HSP90 interacts extensively with a variety of signaling transduction proteins (Whitesell \& Lindquist, 2005),(Mahendrarajah et al., 2017). We and other groups have previously demonstrated that HSP90 could directly interact and regulate STAT3 activation in various cancers and promote cancer cell growth and survival as an oncogene (Prinsloo, Kramer, Edkins \& Blatch, 2012; Schoof, von Bonin, Trumper \& Kube, 2009; Song et al., 2017). However, whether HSP90 is involved in regulating STAT3 activation in skeletal muscles and its functional role in cachectic muscle wasting is still unknown.

Herein, we report that the increased HSP90-STAT3 interaction is necessary to induce prolonged STAT3 activation and muscle atrophy in clinical cachectic patients and C26 tumor-bearing experimental cachexia mice models. Administration of HSP90 inhibitors in vivo could successfully alleviate the pathological development of experimental cachexia; mechanistic study demonstrated that activated STAT3 could induce FOXO1 transcription by binding directly to the FOXO1 promoter, and knockdown of FOXO1 abolished the effects of STAT3 induced muscle wasting. Therefore, our study provided novel experimental evidence showing that the HSP90/STAT3/FOXO1 axis might be a potential therapeutic target for cancer cachexia.

\section{Results}

\section{Enhanced HSP90-STAT3 interaction causes persistent STAT3 activation and muscle atrophy in patients and mice with cancer cachexia.}

We first evaluated the expression level of HSP90, atrophy markers, and activation of STAT3 in muscle from cancer cachexia patients. As shown in Figure 1, there is a significantly increased expression of muscle atrophy markers, including myostatin, E3 ligases such as Atrogin-1 and MuRF1 in skeletal muscle of cancer cachexia patients; also, we detected the persistent activation of STAT3 accompanied by significantly increased expression of HSP90 in cachectic skeletal muscle (Figure 1a,b); by measuring the HSP90-STAT3 interaction using the immunoprecipitation, we observed a dramatic increase in the HSP90-STAT3 interaction in patient muscle with cancer cachexia (Figure 1c), indicating that HSP90 might be involved in the regulation of STAT3 activation during the pathological development of cancer cachexia.

To validate these findings, inoculated Balb/C mice with a mouse colon adenocarcinoma cell line C26 were 
used to establish an experimental cachexia mouse model; these mice showed typical cachectic symptoms, including progressive weight loss, dull and matted fur, and mental weakness, which was consistent with the previous reports (Tanaka et al., 1990). There was approximately $15-20 \%$ overall weight loss and obvious lean body mass weight loss in C26 tumor-bearing mice compared with that in non-tumor-bearing mice within 15 days after tumor inoculation (Figure S1a). Persistent STAT3 activation, as well as the enhanced expression of HSP90 and atrophy related molecular markers including myostatin, Atrogin-1, and MuRF1, were also detected in cachectic muscle from C26-bearing mice (Figure 1d,e), the increase of the HSP90-STAT3 interaction was also confirmed in the cachectic muscle of C26 tumor-bearing mice (Figure 1f).

\section{HSP90 and STAT3 were required for C26 CM-induced myotube atrophy.}

To assess the involvement of HSP90 in cancer cachexia-related muscle wasting, we adopted the in vitro cachectic cell model by culturing the $\mathrm{C} 2 \mathrm{C} 12$ myotubes with $\mathrm{C} 26$ tumor cell-derived conditional medium (C26 CM) (Figure S1b,c). Western blot assay confirms the pathological phenotype of cachectic muscle atrophy based on the loss of MHC expression and the enhanced expression of Atrogin-1, MuRF1, and myostatin (Figure 2a). H\&E staining further demonstrated the myotube shrinking induced by C26 CM (Figure 2c). Notably, we observed a dramatic increase in the HSP90-STAT3 interaction in C2C12 myotubes upon C26 $\mathrm{CM}$ treatment (Figure 2b); this is consistent with the in vivo data, although the increased expression of HSP90 in skeletal muscle was not obvious in C26 CM induced muscle atrophy (Figure 2a). To determine whether the atrophy effect was HSP90-dependent, we knocked down HSP90 in C2C12 myotubes. The results showed that the administration of RNAi against HSP90 or STAT3 prevented the decrease in the myotube size, myotube diameter, and myotube area induced by C26 CM (Figure 2c, d left). These results were further confirmed by the measurement of the transcriptional expression of MuRF-1 and atrogin-1 (Figure 2d right). Consistently, the myotube atrophy-associated phenotypes, including the down-regulated expression of MHC and the up-regulated expression of myostatin, MuRF-1, and atrogin-1, were largely reversed with the knocked down of HSP90 (Figure 2e left). Similar effects were observed when STAT3 was knocked down in C2C12 myotubes (Figure 2e right), indicating that the enhanced HSP90-STAT3 interaction plays a crucial role in the pathological development of cachectic muscle wasting.

\section{Disruption of the HSP90-STAT3 interaction by 17DMAG protected C2C12 myotubes from C26 CM-induced atrophy.}

17DMAG, an inhibitor of HSP90, was then introduced in the $\mathrm{C} 2 \mathrm{C} 12$ myotube atrophy model, which was established by the treatment of $\mathrm{C} 2 \mathrm{C} 12$ myotube with $\mathrm{C} 26$ derived $\mathrm{CM}$; the results showed that 17DMAG treatment prevented myotube shrinking, increased the myotube diameter, and enlarged the myotube area (Figure 3a and b, Figure S2a). Notably, 17DMAG treatment significantly restored MHC expression, decreased the STAT3 activation, and down-regulated the expression of myostatin, MuRF-1, and Atrogin-1 both at the protein and mRNA levels (Figure 3b-d). In addition, the co-expression of MHC, myostatin, and phosphorylated STAT3 (pY-STAT3) was also confirmed by immunofluorescence (Figure 3c). Importantly, 17DMAG disrupted the increase in the binding of HSP90 and JAK2 to STAT3, suggesting that the molecular chaperone activity of HSP90 might prolong the JAK2-STAT3 interaction and STAT3 activation in C26 CM-induced $\mathrm{C} 2 \mathrm{C} 12$ cells (Figure 3e). Thus, we concluded that the 17DMAG treatment protected $\mathrm{C} 2 \mathrm{C} 12$ myotubes from $\mathrm{C} 26 \mathrm{CM}$-induced muscle atrophy and catabolism. In contrast, 17DMAG treatment was not able to ameliorate LPS induced myotube atrophy, suggesting its effect was not TLR4 dependent (Fig S2b).

\section{HSP90 inhibitors ameliorated cachexia in different cachectic mice models.}

To investigate the protective effect of the HSP90 inhibitor in vivo, C26 tumor-bearing cachectic mice were given two HSP90 inhibitors: 17DMAG (15 mg/kg, daily) or PU-H71 (50 mg/kg, daily), respectively. Both drugs were well tolerated by the mice. We observed that the body weights of the mice in the 17DMAG and PU-H71 groups were consistently higher than those in the corresponding vehicle solvent group (Figure 4a, left). And in an additional surviving test, the 17DMAG treatment significantly prolongs the overall survival time compared to its vehicle (shift in the median survival time from 15.5 to 20 days) (Figure

4a, right). However, PU-H71 had only a moderate effect on the survival time, not as significant as that of 
17DMAG (Figure 4a, right). 17DMAG or PU-H71 treatment significantly increased the tumor-free body mass compared with the vehicles (Figure 4d, left). Notably, neither 17DMAG nor PU-H71 treatment inhibited C26 tumor growth, excluding the possibility that the protective effects of 17DMAG or PU-H71 on body weight were based on its antitumor effect (Figure S2c). Since 17DMAG showed greater potential for cachexia treatment than PU-H71, it was chosen for further investigation in our work.

In C26 tumor-bearing mice, the 17DMAG treatment significantly restored grip strength, increased lean body mass, and the mass of four major groups of skeletal muscle tibialis anterior (TA), gastrocnemius (Gastroc), soleus, and extensor digitalis longus (EDL) (Figure 4b-d). In addition, inguinal white adipose tissue (iWAT), which was almost completely absent in C26 tumor-bearing mice, was significantly restored by 17DMAG treatment (Figure S2d). 17DMAG treatment also normalized the downregulated serum triglycerides (TG) levels compared with those in the mock treatment vehicle control. However, 17DMAG did not affect the elevated serum IL-6 level of C26 tumor-bearing mice (Figure S2e).

To validate whether 17DMAG has a universal efficiency in different cancer cachexia models, another cancer cachexia model of Xenograft of LLC (Lewis lung cancer), was applied on C57BL6 mice. As previously reported, the body mass and gastrocnemius muscle mass were decreased in tumor-bearing mice (Gallot et al., 2014). 17DMAG administration significantly attenuated the decrease in body mass and muscle mass (Figure S3a-e), but final tumor weights of 17DMAG treated tumor-bearing mice were significantly reduced compared with tumor-bearing control (Figure S3b), which indicated that 17DMAG not only had a protective effect on cachexia but also inhibited tumor growth in LLC cachectic mice model.

Inhibition of HSP90 attenuated muscle atrophy via disrupted HSP90-STAT3 interaction in C26 and LLC cachectic mice.

Next, we further investigated the protective effect of 17DMAG on cachectic skeletal muscle. H\&E staining and morphometric analysis of the gastrocnemius revealed that C26 tumor-bearing mice exhibited an obvious decrease in muscle fiber size, which was markedly blocked by 17DMAG treatment (Figure 5a). The representative frequency distribution of the myofiber CSA showed a rightward shift in 17DMAG-treated mice (the most frequent value for the myofiber CSA was 1500-2000 ìm²) vs. C26 tumor-bearing mice (the most frequent value for the myofiber CSA was 1000-1500 $\mathrm{im}^{2}$ ) (Figure 5b). The mean CSA of the 17DMAG-treated mice was more than $40 \%$ higher than that of C26 tumor-bearing mice (Figure $5 \mathrm{c}$ ).

We also observed that treatment with 17DMAG prevented the striking loss of MHC protein in C26 tumorbearing mice compared with that in the vehicle control (Figure 5d, e). The significant decreases in STAT3 activation and myostatin, MuRF-1, and Atrogin-1 expression in cachectic muscles in C26 mice indicated that HSP90 blockade could alleviate muscle wasting (Figure 5d). Expression of MuRF1 and Atrogin1 was further confirmed at the transcriptional level (Figure 5f). In support of this data, the increased binding of HSP90 and STAT3 in the muscles of C26 (Figure S4a) and LLC (Figure S3f) cachectic mice was both disrupted by in vivo 17DMAG administration, indicating the HSP90-STAT3 interaction was a crucial event for the induction of cachectic muscle wasting in vivo. To further validate this data, we also repeat the experiments using another HSP90 inhibitor PU-H71, similar results were observed in PU-H71-treated mice or myotubes (Figure S2f).

\section{STAT3-induced myotube atrophy is FOXO1 dependent.}

It has been reported that during muscle atrophy, ubiquitin-proteasome pathway activation and the expression of MuRF-1/Atrogin-1 are regulated by the transcription factor FOXO1 (Lokireddy et al., 2011; Stitt et al., 2004). Therefore, we hypothesized that STAT3 might regulate FOXO1 expression and activation in cachectic skeletal muscle. As shown in Figure 6a, the FOXO1 expression level was significantly increased in C26 cachectic mice muscle, which was down-regulated upon in vivo17DMAG treatment (Figure 6a upper part, Figure S4b), and a similar result was observed in $\mathrm{C} 2 \mathrm{C} 12$ myotubes when it was subjected to $\mathrm{C} 26$ $\mathrm{CM}$ treatment (Figure 6b lower part). The expression of FOXO1 in C26 CM-treated C2C12 myotubes was decreased when STAT3 was knocked down by siRNA (Figure 6b). Next, we transfected a plasmid encoding constitutively activated STAT3 (STAT3-C) (Bromberg et al., 1999) into C2C12 myotubes. As 
expected, STAT3-C transfection significantly up-regulated MuRF-1, Atrogin-1, and myostatin, which was like that observed upon C26 CM treatment. Immunofluorescence staining confirmed that both STAT3$\mathrm{C}$, and $\mathrm{C} 26 \mathrm{CM}$ were able to increase the nuclear expression of FOXO1 (Figure 6c). We next sought to determine whether STAT3 could regulate FOXO1 transcription by direct binding of its promoter. According to the newest version of the JASPAR database (Fornes et al., 2020), two potential STAT3 DNA binding motifs in the promoter region of the FOXO1 gene were predicted (Figure 6d); and validated by chromatin immunoprecipitation assays. As shown in Figure 6e and f, semi-quantitative PCR and qPCR analysis of the DNA immunoprecipitated with the STAT antibody showed the enrichment of the FOXO1 promoter region sequence, indicating direct binding. In contrast, knocking down FOXO1 almost completely abolished STAT3 activation-induced myotube atrophy in both STAT3-C-transfected and C26 CM-treated C2C12 myotube cells (Figure 6g). Our data demonstrated for the first time that in cachectic skeletal muscle, consistent activation of STAT3 could up-regulate ubiquitin-ligases responsible for skeletal muscle protein degradation, MuRF1, and Atrogin1 in a FOXO1 pathway-dependent manner. Notably, the effect of 17DMAG on FOXO1 inhibition was abolished by the overexpression of STAT-C (Figure 6h), confirmed that FOXO1 was regulated by STAT3 at the transcription level.

\section{Discussion}

Muscle wasting is one of the hallmarks of cancer cachexia (Penna, Ballaro, Beltra, De Lucia \& Costelli, 2018). STAT3 signaling plays a crucial role in the regulation of skeletal muscle generation, muscle mass, and repair processes. It is generally believed that transient STAT3 activation in muscles is beneficial to muscle regeneration and hypertrophy, but prolonged STAT3 activation in muscles has been shown to be responsible for muscle wasting (Zimmers, Fishel \& Bonetto, 2016). Increased STAT3 activation has been observed in skeletal muscles in diverse models of cancer cachexia, including models based on IL-6 administration, C26 colon adenocarcinoma, LLC lung carcinoma, B16 melanoma, and ApcMin/+-induced intestinal cancer (Baltgalvis, Berger, Pena, Davis, Muga \& Carson, 2008; Bonetto et al., 2012b; Pretto et al., 2015; Puppa, Gao, Narsale \& Carson, 2014; Silvaet al., 2015). However, the clinical association, the factors triggering abnormal STAT3 activation in cachectic muscles as well as the molecular mechanism of muscle atrophy induced by consistent STAT3 activation still need to be delineated. Here, for the first time, our data confirm the clinical association of abnormal STAT3 activation correlated with the development of cachectic muscle wasting by measuring the biopsy specimens from liver cancer patients.

Most STAT3 molecules exist in the cytoplasm in an unphosphorylated form, and their transcriptional regulatory effects rely on the phosphorylation of the tyrosine 705 residue. The upstream activators of STAT3 include IL-6 family cytokines, growth factors such as EGF, oncogenes such as the Src family kinases, and other biological factors (Yu et al., 1995; Zhong, Wen \& Darnell, 1994; Song et al., 2018). Although the excessive release of IL-6 family cytokines, including IL-6, LIF, OSM, and CNTF, has been linked to the pathological development of cancer cachexia (Fearon, 2013). Multiple studies on STAT3 signaling have demonstrated that the stimulation of non-malignant cells with IL-6 family cytokines can trigger only transient STAT3 activation, which is quickly terminated by negative regulators such as PIAS, suppressor of cytokine signaling (SOCS-1, 2, and 3), and cellular phosphatases (SHP-1/2, DUSP22, PRPRD, PTPRT, and PTPN1/2) (Johnson, O'Keefe \& Grandis, 2018; Ram \& Waxman, 1997; Sengupta, Schmitt \& Ivashkiv, 1996). Therefore, it is reasonable to speculate that apart from IL-6 family cytokines, other regulatory factors may also be involved in maintaining the persistent STAT3 activation in cachectic muscles in cancer.

HSP90 is an ATP-dependent molecular chaperone; it has been reported to be one of the most abundant molecules within cells, accounting for nearly $2 \%$ of all cellular proteins (Joshi, Wang, Araujo, Sharma, Brodsky \& Chiosis, 2018). A wide variety of stressful stimuli, including heat shock, UV radiation, and micro-organismal infections, could promote intracellular HSP90 synthesis. During cancer progression, the expression level of HSP90 increases by 2- to 10-fold in tumor cells (Joshi, Wang, Araujo, Sharma, Brodsky \& Chiosis, 2018). Elevated HSP90 expression has been found in various types of human tumors and is often correlated with increased tumor cell proliferation, inadequate responses to chemotherapy, and poor survival (Kazarian et al., 2017; Ono et al., 2018; Whitesell \& Lindquist, 2005; Yun, Kim, Lim \& Lee, 2019). Over 200 
oncogenic proteins have been identified as clients of HSP90 (Trepel, Mollapour, Giaccone \& Neckers, 2010). We and others have demonstrated a direct interaction between STAT3 and HSP90 in multiple cell types, including cancer cells, epithelial cells, fibroblasts, and endothelial cells (Song et al., 2017; Shah, Patel, Fried \& Sehgal, 2002). Here, in this study, we found an increased interaction of HSP90 and STAT3 in skeletal muscle from cachexia patients and experimental mice model. Administration of HSP90 inhibitors, which blocked the aberrant STAT3 activation, could successfully alleviate cachexia-related muscle wasting phenotype.

Interestingly, we revealed that the direct interaction of HSP90 with STAT3 could enhance JAK2-mediated STAT3 phosphorylation, thus induce muscle atrophy. The increased HSP90-STAT3 interaction likely causes the prolonged STAT3 activation and muscle atrophy since both HSP90 inhibitor or STAT3 siRNA could successfully alleviate the pathological muscle wasting phenotype both in vivo and in vitro ; suggesting that persistent activation of STAT3 rather than other signaling pathway is the major cause of the induction of muscle wasting in cancer cachexia. A previous study by Zhang et al. reported that tumor released HSP90 contained exosome could trigger muscle wasting by activation of TLR4, to validate whether TLR4 mediates the protective effect of HSP90 inhibitors in cachectic muscle wasting, we repeated the experiment by using the LPS induced the $\mathrm{C} 2 \mathrm{C} 12$ myotube atrophy model, however, to our surprise, we did not observe the obvious protective effects; this result suggested that the protective effect of HSP90 inhibitors in cachectic muscle wasting is not fully TLR4 dependent. TLR4 signaling might be involved in the initial stage of STAT3 activation, but the tumor induced a prolonged STAT3 activation, which is necessary for triggering the muscle wasting as demonstrated in our mechanism study by over-expression of the consistent activated form of STAT3 (STAT3-C) in C2C12 myotube, required the participation of HSP90 as a molecular chaperone by the interaction of STAT3. Therefore, it is reasonable to speculate that the enhanced HSP90-STAT3 interaction seems to be the critical event for the induction of persistent STAT3 activation and cachectic muscle atrophy.

An additional goal of this study was to delineate the mechanism by which the prolonged STAT3 activation induces muscle wasting. The pathogenesis of muscle wasting in cancer cachexia has been demonstrated to be the result of an imbalance between the anabolic and catabolic pathways. The STAT3 pathway has been found to be a crucial pathway involved in this process. During the catabolic response in skeletal muscles, prolonged STAT3 activation could disturb the sensitivity of the insulin/IGF-1/AKT signaling pathway by enhancing the expression of inhibitory SOCS proteins (Mashili, Chibalin, Krook \& Zierath, 2013; Ueki, Kondo \& Kahn, 2004). During the anabolic response, current models indicate that STAT3 could enhance the expression of myostatin via activation of CAAT/enhancer-binding protein (C/EBP) (Zhang et al., 2013). Myostatin is a TGF-beta family cytokine that can repress muscle growth and disturb myogenesis. Our study observed the elevated expression of myostatin accompanied by prolonged STAT3 activation, which fully supports our conclusion. However, the most interesting mechanism revealed by our study is that the prolonged activation of STAT3 in skeletal muscle derived from either C26 tumor-bearing cachectic mice or $\mathrm{C} 2 \mathrm{C} 12$ myotubes treated with $\mathrm{C} 26 \mathrm{CM}$ was correlated with elevated expression of UPS-related muscle-specific E3 ubiquitin ligases, such as Atrogin-1 and MuRF1. In addition, the transfection-induced overexpression of constitutively activated STAT3 (STAT3-C) also triggered E3 ligases, indicating that constitutive activation of STAT3 itself might be enough to induce muscle wasting by activating E3 ligases mediated protein degradation pathways. However, the pathway linking STAT3 and the activation of the protein degradation pathway has never been evaluated.

FOXO1, as a key member of the forkhead box protein O (FOXO) transcription factor family, has been found to be widely expressed in various types of metabolism-associated organs, such as the liver and skeletal muscles. In skeletal muscles, the FOXO1 transcription factor represents a master regulator of muscle growth, regeneration, and atrophy. Increased FOXO1 expression and activation have been found in skeletal muscle in energy-deprived states such as fasting, cancer, and severe diabetes (O'Neill et al., 2019; Reed, Sandesara, Senf \& Judge, 2012). Enhanced FOXO1 activation in skeletal muscle triggered the muscle atrophy by activation of the UPS and lysosomal proteolysis through the upregulation of FOXO downstream target genes, including atrogin-1 and MuRF1 (Milan et al., 2015). Genetic data demonstrated that STAT3 expression and activation are correlated with the intact function of FOXO transcription factors; however, whether FOXO1 is necessary for STAT3-induced muscle wasting or vice versa is still not known. In this study, we demonstrated for the 
first time that STAT3 could regulate the expression and nuclear translocation of FOXO1 in the skeletal muscle, subsequently up-regulated E3 ligases and FOXO1-dependent triggering of muscle wasting, which could partly explain the pathological mechanism by which consistent STAT3 activation in the skeletal muscle could trigger muscle atrophy. Since the bioinformatics study demonstrated that there are several STAT3 consensus motifs in FOXO1 promoter regions, our subsequent ChIP analysis indicated that STAT3 could bind to the FOXO1 promoter and directly regulate the FOXO1 gene transcription in skeletal muscles. To test the pathogenic effects of STAT3 on muscle atrophy, a constitutively active recombinant STAT3 mutant expression plasmid, STAT3-C, was adopted in our study. Our results showed that the overexpression of the constitutively activated form of STAT3 in skeletal muscle could mimic the effect of the C26 cell-conditioned medium on muscle wasting phenotype induction. Knocking down the expression of FOXO1 in STAT3-Ctransfected or C26 cell-conditioned medium-treated myotubes almost completely abolished the activation of the ubiquitin-proteasome system and eliminated muscle wasting, suggesting that STAT3 activation-induced muscle atrophy is indeed FOXO1 dependent.

In summary, our study demonstrated that HSP90 inhibitors could effectively alleviate cancer cachexiainduced skeletal muscle wastingin vivo ; these effects might be associated with the abnormal activation of the JAK2/STAT3/FOXO1 and ubiquitin-proteasome pathways. The close monitoring of HSP90 levels in cancer patients and the development of strategies to block HSP90 activity could potentially prevent or reverse cachexia development.

\section{Materials and Methods}

\section{Cell culture}

The colon 26 murine adenocarcinoma cell line (C26) and Mouse colon carcinoma (CT26) cells were cultured as a monolayer in RPMI-1640 medium (Life Technology, NY) supplemented with 10\% fetal bovine serum, L-glutamine, and 100 units/ml penicillin and streptomycin (HyClone, Logan, UT in a humidified atmosphere containing $5 \% \mathrm{CO} 2$ at $37{ }^{\circ} \mathrm{C}$. Murine $\mathrm{C} 2 \mathrm{C} 12$ myoblasts obtained from the American Type Culture Collection (ATCC) were maintained in Dulbecco's modified Eagle medium (DMEM) supplemented with $10 \%$ fetal bovine serum and antibiotics (100 U/ml penicillin and streptomycin; referred to as growth medium, GM). To induce myotube differentiation, cells were grown to $100 \%$ confluence and exposed to DMEM containing $2 \%$ horse serum (Life Technology, NY) (referred to as differentiation medium, DM) for up to 4 days.

\section{Mouse model of cachexia}

Six-week-old male BALB/C mice (obtained from the Model Animal Research Center of Nanjing University) were maintained under specific pathogen-free (SPF) conditions and allowed to acclimate for one week. Mice were housed in individually ventilated cages (IVCs) with free access to drinking water and a basal diet under controlled conditions of humidity, light ( $12 \mathrm{~h}$ light/12 h dark cycle), and temperature.

To induce the cachexia mouse model, $100 \mu \mathrm{l}$ C26 murine adenocarcinoma cells were subcutaneously inoculated into the right flanks of BALB/C mice $\left(5 \times 10^{5}\right.$ cells/mouse). When the mice lost $20 \%$ of their body weight or their tumor volumes reached $1500 \mathrm{~mm}^{3}$, the mice were sacrificed. Tumors, muscles, and other organs, such as the liver and hypothalamus, were rapidly dissected, frozen in liquid nitrogen and stored at $-80^{\circ} \mathrm{C}$ until further analyses or fixed with $10 \%$ formaldehyde for immunohistological staining. All experiments involving animals were conducted according to protocols approved by the Institutional Animal Care and Use Committee of Nanjing University, in accordance with the guidelines for animal care and use of Jiangsu Province.

\section{Patients' biopsy specimens}

The study was approved by the Ethics Committee in the Medical School of Nanjing University (IRB no.: 20200115003). All patients provided written informed consent. Cachexia was defined clinically as documented non-intentional dry weight loss of $¿ 5 \mathrm{~kg}$ (all $¿ 10 \%$ of their previous normal weight) at least in [?]3 months (10). All cachectic patients also complained of weight loss; patients' skeletal biopsy specimens of abdominal muscles were obtained after hepatobiliary surgery. Control samples were acquired from patients undergoing elective orthopedic surgery. The information details of patients were listed in Table S1. 


\section{In vivo experiments and tissue collection}

To examine the protective effects of the HSP90 inhibitor on cachexia, the cachectic mice received intraperitoneal injection of 17DMAG (HY-12024,MCE,NJ) $(15 \mathrm{mg} / \mathrm{kg}$, daily) dissolved in 1\% DMSO + 30\% PEG300 + 1\% Tween 80 or PU-H71 (HY11038,MCE,NJ) (50 mg/kg, daily) dissolved in PBS (as the solvent control) beginning on day 7 when the tumor became palpable; vehicle group were injected with relevant solvent: 17DMAG solvent (1\% DMSO + 30\% PEG300 + 1\% Tween 80) for veh1 and PU-H71 solvent (PBS) for veh2. For the survival experiment, fifty mice were randomly divided into five groups (normal control, C26 + 17DMAG, C26+veh1, C26+PU-H71, C26+veh2), and each group contained 10 mice. The mice were followed until the endpoint criterion was fulfilled or at least 30 days had elapsed after the inoculation of C26 cells to determine the survival rate. The endpoint criterion combined the detected weight loss with the overall condition of the mouse. To evaluate the overall health status of the mice, the following aspects were considered in addition to weight loss: appearance, posture, and natural and provoked behaviour (inactivity, dyskinesia, and reduced response to external stimuli). Mice were euthanized when two researchers confirmed the fulfilment of the endpoint criteria.

To study the potential mechanisms involved, another experiment was conducted with a predetermined endpoint at day 15 after $\mathrm{C} 26$ cell inoculation. The numbers of mice and groups were the same as those used in the survival experiment. The following data were collected: body weight, food intake, tumor weight, grip strength, and survival rate. Once the tumors were removed, the gastrocnemius was dissected, harvested, weighed, and then fixed in 4\% paraformaldehyde (Sigma-Aldrich, St. Louis, MO) for subsequent histological analysis.

\section{Assessment of grip strength}

Skeletal muscular strength in mice was quantified with the grip strength test. At the end of the experiment, the forelimb grip strength of each of the four limbs was measured with a Digital Grip Strength Metre (Nanjing Biomedical Research Institute of Nanjing University). Mice were held by the tail and allowed to grasp a grid with their forepaws or with all four paws. The mice were gently pulled by the tail until they released their grip. Five measurements at 3 min intervals were recorded, and the average value for each mouse was determined.

\section{Histological analysis and immunofluorescence}

Procedures for the determination of the fiber cross-sectional area (CSA) were conducted as previously described (Park et al., 2013). Briefly, fixed transverse sections $(7 \mu \mathrm{m})$ were cut from the mid-belly of the gastrocnemius with a cryostat at $-20^{\circ} \mathrm{C}$ and then stained with hematoxylin and eosin $(\mathrm{H} \& \mathrm{E})$. Images were obtained at 40× magnification and quantified using ImageJ software (National Institutes of Health, Bethesda, MD). The CSA and number of myofibers with central nuclei among the individual myofibers were determined by using ImageJ 1.48 software in five random fields for each section.

For immunofluorescence analysis, cells were seeded onto sterile preprocessed glass coverslips that were precoated with $1 \%$ gelatin. After $\mathrm{C} 2 \mathrm{C} 12$ myoblasts had differentiated into myotubes, the cells were washed twice with PBS, followed by fixation in $4 \%$ paraformaldehyde for $15 \mathrm{~min}$. After being rehydrated in PBS, the cells were blocked for $30 \mathrm{~min}$ in $1 \%$ bovine serum albumin (BSA) in PBS containing $0.2 \%$ Triton-X (PBST). Then, the cells were incubated with an anti-myosin heavy chain (MHC) (\#MAB4470, R\&D Systems,MN) (1:100) in $1 \%$ BSA/PBST overnight at $4 \mathrm{degC}$. Cells were then incubated with a fluorescence-labeled secondary anti-mouse antibody $(1: 1000)$ and DAPI (1:1000) at room temperature for $1 \mathrm{~h}$. The specimens were examined under an FV10i laser scanning confocal microscope (Olympus, Tokyo, Japan).

\section{Western blot and immunoprecipitation assays}

Tissues and cell proteins were extracted in ice-cold RIPA lysis buffer consisting of $150 \mathrm{mM} \mathrm{NaCl}, 50 \mathrm{mM}$ Tris-HCl, $0.5 \%$ sodium deoxycholate, $200 \mathrm{mM} \mathrm{NaF}, 200 \mathrm{mM}$ PMSF, 1.0\% NP40, $1 \mathrm{mM}$ EDTA, and protease and phosphatase inhibitor cocktail (Life Technology, NY). The protein concentration in the supernatants 
was determined with a BCA Kit (Pierce, Rockford, IL). Then, $20 \mu \mathrm{g}$ of total protein was separated by electrophoresis in a 10\% SDS-polyacrylamide gel and transferred to a PVDF membrane (Millipore, Billerica, MA) for western blot analysis. The signals were detected with the following antibodies by following standard procedures: STAT3 (\#9145,CST, MA), phosphorylated STAT3 (\#9139,CST, MA), HSP90 (ab13492, Abcam, MA), myostatin (ab71808, Abcam,MA), CD63 (ab8219,Abcam, MA), atrogin-1 (sc166806,Santa Cruz, MA), MuRF-1 (sc398608,Santa Cruz, TX), MHC (\#MAB4470,R\&D Systems, MN), GAPDH (ab181602,Abcam, MA).

For the immunoprecipitation assays, cells were lysed in WB/IP buffer, and proteins were immunoprecipitated with the indicated antibodies. Precleared Protein A/G Plus-Agarose beads (Life Technology, New York, NY) were incubated with the immunocomplexes overnight and washed three times with lysis buffer. The immunoprecipitates were subjected to SDS-PAGE followed by immunoblotting.

\section{Transfection of small interfering RNAs (siRNAs) and overexpression plasmids}

siRNA transfection was performed using Lipofectamine RNAiMAX transfection reagent (Life Technology, New York, NY) by following the manufacturer's guidelines. Briefly, C2C12 cells were seeded into a six-well plate. After four days of differentiation, 5 pmol of siRNA was diluted with Opti-MEM and mixed with the transfection reagent, and the mixture was added to each well. After transfection for $24 \mathrm{~h}$, the cells were challenged with different treatments; some were co-cultured with $\mathrm{C} 26$ cell-conditioned media (C26 $\mathrm{CM}$ ), and some were cultured in DMEM. The siRNAs specific to HSP90aa1 were purchased from Thermo (\#159050,Life Technology, New York, NY). The siRNAs specific to FOXO1 were purchased from Santa Cruz (\#56458,Santa Cruz, TX).

The constitutively activated STAT3 plasmids (STAT3-C Flag pRc/CMV) were purchased from Addgene (Plasmid \#8722). C2C12 cells were transiently transfected with STAT3-C plasmids using the Lipofectamine 2000 Transfection Reagent according to the manufacturer's instructions (Life Technology, New York, NY).

\section{Chromatin immunoprecipitation (ChIP) analysis}

The binding site of STAT3 to the promoter region of FOXO1 is predicted by CiiiDER software according to the JASPAR database (Fornes et al., 2020; Gearing et al., 2019). The ChIP assay was performed according to the manufacturer's instructions (P2078,Beyotime,China). C2C12 cells were fixed with $1 \%$ formaldehyde at $37^{\circ} \mathrm{C}$ for $10 \mathrm{~min}$, lysed, and sonicated. Soluble chromatin was coimmunoprecipitated with anti-STAT3 antibodies or an equal amount of rabbit IgG. The immunoprecipitated and input DNA was subjected to PCR and qPCR using FOXO1 promoter site-specific primers (5'-CGACTTCAACACCTCATCGCTTC-3' and 5'AGGCGCGCAGATCCTTCGGTGA-3'). Q-PCR was performed with Power SYBR Green PCR Master Mix (Life Technologies, New York, NY). The fold change in enrichment relative to the input DNA was calculated using the comparative $\mathrm{Ct}$ method ([?][?]Ct).

\section{Real-time PCR assay}

Total RNA from either snap-frozen muscles or cultured cells was isolated using an RNeasy Micro Kit (Qiagen, Hilden, Germany). The mRNA was reverse-transcribed into cDNA with PrimeScript RT Master Mix (TaKaRa, Otsu, Japan) and subjected to quantitative real-time PCR with SYBR Green PCR Master Mix (Life Technologies,New York,NY). The relative expression of the target genes was normalized to that of GAPDH and calculated with the $\Delta \Delta$ Ct method. The following primers were used: MuRF-1: forward: 5'ACCTGCTGGTGGAAAACATC-3', reverse: 5'-AGGAGCAAGTAGGCACCTCA-3'; Atrogin-1: forward: 5'-ATTCTACACTGGCAGCAGCA-3', reverse: 5'-TCAGCCTCTGCATGATGTTC-3'; GAPDH: forward: 5'-ACCCTTAAGAGGGATGCTGC-3', reverse: 5'-CGGGACGAGGAAACACTCTC-3'.

\section{Statistical analysis}

The tissue weights of different groups were compared using Student's t-test. Longitudinal body weight or muscle mass differences were analyzed by repeated measurement analysis of variance (ANOVA). The 
survival rate difference was determined with the chi-square test. $\mathrm{P}$ values $<0.05$ were considered statistically significant.

\section{Author contributions}

M.N, S.S, and Z.S designed and performed the experiments, analyzed data, and drafted the manuscript. W. $\mathrm{P}$ and C.Z. performed a part of the animal experiments. L.W, L.L, and Y.D performed a part of the in vitroexperiments. W.C, Q. G, and H.W conceived the projects, designed the experiments, analyzed data, and wrote the manuscript. All authors read and approved the final manuscript.

\section{Conflict of interest}

The authors declare no conflict of interest.

\section{Acknowledgments}

This work was supported by grants from the National Natural Science Foundation of China (Nos. 82070912, 81773326, and 81471095), the Key Project of Research and Development of Ningxia Hui Autonomous Region of China (No. 2017BN04), a grant from the Natural Science Foundation of Jiangsu Province China (No. BK20171347 and BE2019676) and the Qing Lan Project of Jiangsu Province China. We thank Prof Erguang Li for his critical review and thoughtful discussion of the manuscript.

\section{REFERENCES}

Baltgalvis, K.A., Berger, F.G., Pena, M.M., Davis, J.M., Muga, S.J., \& Carson, J.A. (2008). Interleukin6 and cachexia in ApcMin/+ mice.Am J Physiol Regul Integr Comp Physiol . 294(2), R393-R401. 10.1152/ajpregu.00716.2007

Bilodeau, P.A., Coyne, E.S., \& Wing, S.S. (2016). The ubiquitin proteasome system in atrophying skeletal muscle: roles and regulation.Am J Physiol Cell Physiol . 311(3), C392-C403. 10.1152/ajpcell.00125.2016

Bodine, S.C., Latres, E., Baumhueter, S., Lai, V.K., Nunez, L., \& Clarke, B.A., et al. (2001). Identification of ubiquitin ligases required for skeletal muscle atrophy. Science . 294(5547), 1704-1708. 10.1126/science.1065874

Bonetto, A., Aydogdu, T., Jin, X., Zhang, Z., Zhan, R., \& Puzis, L., et al. (2012a). JAK/STAT3 pathway inhibition blocks skeletal muscle wasting downstream of IL-6 and in experimental cancer cachexia. Am J Physiol Endocrinol Metab . 303(3), E410-E421. 10.1152/ajpendo.00039.2012

Bonetto, A., Aydogdu, T., Jin, X., Zhang, Z., Zhan, R., \& Puzis, L., et al. (2012b). JAK/STAT3 pathway inhibition blocks skeletal muscle wasting downstream of IL-6 and in experimental cancer cachexia. Am $J$ Physiol Endocrinol Metab . 303(3), E410-E421. 10.1152/ajpendo.00039.2012

Bromberg, J.F., Wrzeszczynska, M.H., Devgan, G., Zhao, Y., Pestell, R.G., Albanese, C., \& Darnell, J.J. (1999). Stat3 as an oncogene. Cell . 98(3), 295-303. 10.1016/s0092-8674(00)81959-5

Fearon, K.H. (2013). Selective androgen receptor modulators in cancer cachexia? Lancet Oncol . 14(4), 271-272. 10.1016/S1470-2045(13)70068-8

Fornes, O., Castro-Mondragon, J.A., Khan, A., van der Lee, R., Zhang, X., \& Richmond, P.A., et al. (2020). JASPAR 2020: update of the open-access database of transcription factor binding profiles.Nucleic Acids Res . 48(D1), D87-D92. 10.1093/nar/gkz1001

Gallot, Y.S., Durieux, A., Castells, J., Desgeorges, M.M., Vernus, B., \& Plantureux, L., et al. (2014). Myostatin Gene Inactivation Prevents Skeletal Muscle Wasting in Cancer. Cancer Research . 74(24), 73447356. 10.1158/0008-5472.CAN-14-0057

Gearing, L.J., Cumming, H.E., Chapman, R., Finkel, A.M., Woodhouse, I.B., \& Luu, K., et al. (2019). CiiiDER: A tool for predicting and analysing transcription factor binding sites. PLoS One . 14(9), e215495. 10.1371/journal.pone.0215495 
Guadagnin, E., Mazala, D., \& Chen, Y.W. (2018). STAT3 in Skeletal Muscle Function and Disorders. Int J Mol Sci . 19(8). 10.3390/ijms19082265

Guo, D., Wang, C., Wang, Q., Qiao, Z., \& Tang, H. (2017). Pantoprazole blocks the JAK2/STAT3 pathway to alleviate skeletal muscle wasting in cancer cachexia by inhibiting inflammatory response. Oncotarget . 8(24), 39640-39648. 10.18632/oncotarget.17387

Johnson, D.E., O'Keefe, R.A., \& Grandis, J.R. (2018). Targeting the IL-6/JAK/STAT3 signalling axis in cancer. Nat Rev Clin Oncol . 15(4), 234-248. 10.1038/nrclinonc.2018.8

Joshi, S., Wang, T., Araujo, T., Sharma, S., Brodsky, J.L., \& Chiosis, G. (2018). Adapting to stress chaperome networks in cancer. Nat Rev Cancer . 18(9), 562-575. 10.1038/s41568-018-0020-9

Kazarian, A., Blyuss, O., Metodieva, G., Gentry-Maharaj, A., Ryan, A., \& Kiseleva, E.M., et al. (2017). Testing breast cancer serum biomarkers for early detection and prognosis in pre-diagnosis samples.British Journal of Cancer . 116(4), 501-508. 10.1038/bjc.2016.433

Lokireddy, S., Mouly, V., Butler-Browne, G., Gluckman, P.D., Sharma, M., Kambadur, R., \& McFarlane, C. (2011). Myostatin promotes the wasting of human myoblast cultures through promoting ubiquitinproteasome pathway-mediated loss of sarcomeric proteins. Am J Physiol Cell Physiol . 301(6), C1316-C1324. 10.1152/ajpcell.00114.2011

Ma, J.F., Sanchez, B.J., Hall, D.T., Tremblay, A.K., Di Marco, S., \& Gallouzi, I.E. (2017). STAT3 promotes IFNgamma/TNFalpha-induced muscle wasting in an NF-kappaB-dependent and IL-6-independent manner.EMBO Mol Med . 9(5), 622-637. 10.15252/emmm.201607052

Mahendrarajah, N., Borisova, M.E., Reichardt, S., Godmann, M., Sellmer, A., \& Mahboobi, S., et al. (2017). HSP90 is necessary for the ACK1-dependent phosphorylation of STAT1 and STAT3. Cell Signal . 39, 9-17. 10.1016/j.cellsig.2017.07.014

Mashili, F., Chibalin, A.V., Krook, A., \& Zierath, J.R. (2013). Constitutive STAT3 phosphorylation contributes to skeletal muscle insulin resistance in type 2 diabetes. Diabetes . 62(2), 457-465. 10.2337/db12-0337

Milan, G., Romanello, V., Pescatore, F., Armani, A., Paik, J.H., \& Frasson, L., et al. (2015). Regulation of autophagy and the ubiquitin-proteasome system by the FoxO transcriptional network during muscle atrophy. Nat Commun . 6, 6670. 10.1038/ncomms7670

Mubaid, S., Ma, J.F., Omer, A., Ashour, K., Lian, X.J., \& Sanchez, B.J., et al. (2019). HuR counteracts miR-330 to promote STAT3 translation during inflammation-induced muscle wasting. Proc Natl Acad Sci U S A . 116(35), 17261-17270. 10.1073/pnas.1905172116

O’Neill, B.T., Bhardwaj, G., Penniman, C.M., Krumpoch, M.T., Suarez, B.P., \& Klaus, K., et al. (2019). FoxO Transcription Factors Are Critical Regulators of Diabetes-Related Muscle Atrophy. Diabetes . 68(3), 556-570. $10.2337 / \mathrm{db} 18-0416$

Ono, K., Eguchi, T., Sogawa, C., Calderwood, S.K., Futagawa, J., \& Kasai, T., et al. (2018). HSP-enriched properties of extracellular vesicles involve survival of metastatic oral cancer cells. J Cell Biochem . 119(9), 7350-7362. $10.1002 /$ jcb.27039

Park, B.S., Henning, P.C., Grant, S.C., Lee, W.J., Lee, S.R., Arjmandi, B.H., \& Kim, J.S. (2013). HMB attenuates muscle loss during sustained energy deficit induced by calorie restriction and endurance exercise.Metabolism . 62(12), 1718-1729. 10.1016/j.metabol.2013.06.005

Penna, F., Ballaro, R., Beltra, M., De Lucia, S., \& Costelli, P. (2018). Modulating Metabolism to Improve Cancer-Induced Muscle Wasting. Oxid Med Cell Longev . 2018, 7153610. 10.1155/2018/7153610

Pretto, F., Ghilardi, C., Moschetta, M., Bassi, A., Rovida, A., \& Scarlato, V., et al. (2015). Sunitinib prevents cachexia and prolongs survival of mice bearing renal cancer by restraining STAT3 and MuRF-1 activation in muscle. Oncotarget . 6(5), 3043-3054. 10.18632/oncotarget.2812 
Prinsloo, E., Kramer, A.H., Edkins, A.L., \& Blatch, G.L. (2012). STAT3 interacts directly with Hsp90. IUBMB Life . 64(3), 266-273. 10.1002/iub.607

Puppa, M.J., Gao, S., Narsale, A.A., \& Carson, J.A. (2014). Skeletal muscle glycoprotein 130's role in Lewis lung carcinoma-induced cachexia.FASEB J . 28(2), 998-1009. 10.1096/fj.13-240580

Ram, P.A., \& Waxman, D.J. (1997). Interaction of growth hormone-activated STATs with SH2-containing phosphotyrosine phosphatase SHP-1 and nuclear JAK2 tyrosine kinase. J Biol Chem . 272(28), 17694-17702. $10.1074 / \mathrm{jbc} .272 .28 .17694$

Reed, S.A., Sandesara, P.B., Senf, S.M., \& Judge, A.R. (2012). Inhibition of FoxO transcriptional activity prevents muscle fiber atrophy during cachexia and induces hypertrophy. FASEB J . 26(3), 987-1000. 10.1096/fj.11-189977

Schoof, N., von Bonin, F., Trumper, L., \& Kube, D. (2009). HSP90 is essential for Jak-STAT signaling in classical Hodgkin lymphoma cells. Cell Commun Signal . 7, 17. 10.1186/1478-811X-7-17

Schopf, F.H., Biebl, M.M., \& Buchner, J. (2017). The HSP90 chaperone machinery. Nature Reviews Molecular Cell Biology . 18(6), 345-360. 10.1038/nrm.2017.20

Sengupta, T.K., Schmitt, E.M., \& Ivashkiv, L.B. (1996). Inhibition of cytokines and JAK-STAT activation by distinct signaling pathways.Proc Natl Acad Sci U S A . 93(18), 9499-9504. 10.1073/pnas.93.18.9499

Shah, M., Patel, K., Fried, V.A., \& Sehgal, P.B. (2002). Interactions of STAT3 with caveolin-1 and heat shock protein 90 in plasma membrane raft and cytosolic complexes. Preservation of cytokine signaling during fever. J Biol Chem . 277(47), 45662-45669. 10.1074/jbc.M205935200

Silva, K.A., Dong, J., Dong, Y., Dong, Y., Schor, N., \& Tweardy, D.J., et al. (2015). Inhibition of Stat3 activation suppresses caspase-3 and the ubiquitin-proteasome system, leading to preservation of muscle mass in cancer cachexia. J Biol Chem . 290(17), 11177-11187. 10.1074/jbc.M115.641514

Song, S., Min, H., Niu, M., Wang, L., Wu, Y., \& Zhang, B., et al. (2018). S1PR1 predicts patient survival and promotes chemotherapy drug resistance in gastric cancer cells through STAT3 constitutive activation. EBioMedicine . 37, 168-176. 10.1016/j.ebiom.2018.10.005

Song, S., Su, Z., Xu, H., Niu, M., Chen, X., \& Min, H., et al. (2017). Luteolin selectively kills STAT3 highly activated gastric cancer cells through enhancing the binding of STAT3 to SHP-1. Cell Death 8 Disease . 8(2), e2612. 10.1038/cddis.2017.38

Stitt, T.N., Drujan, D., Clarke, B.A., Panaro, F., Timofeyva, Y., \& Kline, W.O., et al. (2004). The IGF-1/PI3K/Akt Pathway Prevents Expression of Muscle Atrophy-Induced Ubiquitin Ligases by Inhibiting FOXO Transcription Factors. Molecular Cell . 14(3), 395-403. https://doi.org/10.1016/S10972765(04)00211-4

Tanaka, Y., Eda, H., Tanaka, T., Udagawa, T., Ishikawa, T., \& Horii, I., et al. (1990). Experimental cancer cachexia induced by transplantable colon 26 adenocarcinoma in mice. Cancer Res . 50(8), 2290-2295.

Trepel, J., Mollapour, M., Giaccone, G., \& Neckers, L. (2010). Targeting the dynamic HSP90 complex in cancer. Nat Rev Cancer . 10(8), 537-549. 10.1038/nrc2887

Ueki, K., Kondo, T., \& Kahn, C.R. (2004). Suppressor of cytokine signaling 1 (SOCS-1) and SOCS-3 cause insulin resistance through inhibition of tyrosine phosphorylation of insulin receptor substrate proteins by discrete mechanisms. Mol Cell Biol . 24(12), 5434-5446. 10.1128/MCB.24.12.5434-5446.2004

Whitesell, L., \& Lindquist, S.L. (2005). HSP90 and the chaperoning of cancer. Nature Reviews Cancer . 5(10), 761-772. 10.1038/nrc1716

Yu, C.L., Meyer, D.J., Campbell, G.S., Larner, A.C., Carter-Su, C., Schwartz, J., \& Jove, R. (1995). Enhanced DNA-binding activity of a Stat3-related protein in cells transformed by the Src oncoprotein.Science 
. 269(5220), 81-83. 10.1126/science.7541555

Yun, C.W., Kim, H.J., Lim, J.H., \& Lee, S.H. (2019). Heat Shock Proteins: Agents of Cancer Development and Therapeutic Targets in Anti-Cancer Therapy. Cells . 9(1). 10.3390/cells9010060

Zhang, L., Pan, J., Dong, Y., Tweardy, D.J., Dong, Y., Garibotto, G., \& Mitch, W.E. (2013). Stat3 activation links a C/EBPdelta to myostatin pathway to stimulate loss of muscle mass. Cell Metab . 18(3), 368-379. 10.1016/j.cmet.2013.07.012

Zhong, Z., Wen, Z., \& Darnell, J.J. (1994). Stat3: a STAT family member activated by tyrosine phosphorylation in response to epidermal growth factor and interleukin-6. Science . 264(5155), 95-98. 10.1126/science. 8140422

Zimmers, T.A., Fishel, M.L., \& Bonetto, A. (2016). STAT3 in the systemic inflammation of cancer cachexia. Seminars in Cell \& Developmental Biology . 54, 28-41. 10.1016/j.semcdb.2016.02.009

Mashili, F., Chibalin, A.V., Krook, A., \& Zierath, J.R. (2013). Constitutive STAT3 phosphorylation contributes to skeletal muscle insulin resistance in type 2 diabetes. Diabetes . 62(2), 457-465. 10.2337/db12-0337

Milan, G., Romanello, V., Pescatore, F., Armani, A., Paik, J.H., \& Frasson, L., et al. (2015). Regulation of autophagy and the ubiquitin-proteasome system by the FoxO transcriptional network during muscle atrophy.Nat Commun . 6, 6670. 10.1038/ncomms7670

Mubaid, S., Ma, J.F., Omer, A., Ashour, K., Lian, X.J., \& Sanchez, B.J., et al. (2019). HuR counteracts miR-330 to promote STAT3 translation during inflammation-induced muscle wasting. Proc Natl Acad Sci U $S$ A . 116(35), 17261-17270. 10.1073/pnas.1905172116

O'Neill, B.T., Bhardwaj, G., Penniman, C.M., Krumpoch, M.T., Suarez, B.P., \& Klaus, K., et al. (2019). FoxO Transcription Factors Are Critical Regulators of Diabetes-Related Muscle Atrophy. Diabetes . 68(3), 556-570. 10.2337/db18-0416

Ono, K., Eguchi, T., Sogawa, C., Calderwood, S.K., Futagawa, J., \& Kasai, T., et al. (2018). HSP-enriched properties of extracellular vesicles involve survival of metastatic oral cancer cells. J Cell Biochem . 119(9), 7350-7362. 10.1002/jcb.27039

Park, B.S., Henning, P.C., Grant, S.C., Lee, W.J., Lee, S.R., Arjmandi, B.H., \& Kim, J.S. (2013). HMB attenuates muscle loss during sustained energy deficit induced by calorie restriction and endurance exercise.Metabolism . 62(12), 1718-1729. 10.1016/j.metabol.2013.06.005

Penna, F., Ballaro, R., Beltra, M., De Lucia, S., \& Costelli, P. (2018). Modulating Metabolism to Improve Cancer-Induced Muscle Wasting. Oxid Med Cell Longev . 2018, 7153610. 10.1155/2018/7153610

Pretto, F., Ghilardi, C., Moschetta, M., Bassi, A., Rovida, A., \& Scarlato, V., et al. (2015). Sunitinib prevents cachexia and prolongs survival of mice bearing renal cancer by restraining STAT3 and MuRF-1 activation in muscle. Oncotarget . 6(5), 3043-3054. 10.18632/oncotarget.2812

Prinsloo, E., Kramer, A.H., Edkins, A.L., \& Blatch, G.L. (2012). STAT3 interacts directly with Hsp90. IUBMB Life . 64(3), 266-273. 10.1002/iub.607

Puppa, M.J., Gao, S., Narsale, A.A., \& Carson, J.A. (2014). Skeletal muscle glycoprotein 130's role in Lewis lung carcinoma-induced cachexia.FASEB J . 28(2), 998-1009. 10.1096/fj.13-240580

Ram, P.A., \& Waxman, D.J. (1997). Interaction of growth hormone-activated STATs with SH2-containing phosphotyrosine phosphatase SHP-1 and nuclear JAK2 tyrosine kinase. J Biol Chem . 272(28), 17694-17702. $10.1074 / \mathrm{jbc} .272 .28 .17694$

Reed, S.A., Sandesara, P.B., Senf, S.M., \& Judge, A.R. (2012). Inhibition of FoxO transcriptional activity prevents muscle fiber atrophy during cachexia and induces hypertrophy. FASEB J . 26(3), 987-1000. 10.1096/fj.11-189977 
Schoof, N., von Bonin, F., Trumper, L., \& Kube, D. (2009). HSP90 is essential for Jak-STAT signaling in classical Hodgkin lymphoma cells. Cell Commun Signal . 7, 17. 10.1186/1478-811X-7-17

Schopf, F.H., Biebl, M.M., \& Buchner, J. (2017). The HSP90 chaperone machinery.Nature Reviews Molecular Cell Biology . 18(6), 345-360. 10.1038/nrm.2017.20

Sengupta, T.K., Schmitt, E.M., \& Ivashkiv, L.B. (1996). Inhibition of cytokines and JAK-STAT activation by distinct signaling pathways. Proc Natl Acad Sci U S A . 93(18), 9499-9504. 10.1073/pnas.93.18.9499

Shah, M., Patel, K., Fried, V.A., \& Sehgal, P.B. (2002). Interactions of STAT3 with caveolin-1 and heat shock protein 90 in plasma membrane raft and cytosolic complexes. Preservation of cytokine signaling during fever. J Biol Chem . 277(47), 45662-45669. 10.1074/jbc.M205935200

Silva, K.A., Dong, J., Dong, Y., Dong, Y., Schor, N., \& Tweardy, D.J., et al. (2015). Inhibition of Stat3 activation suppresses caspase-3 and the ubiquitin-proteasome system, leading to preservation of muscle mass in cancer cachexia. J Biol Chem . 290(17), 11177-11187. 10.1074/jbc.M115.641514

Song, S., Min, H., Niu, M., Wang, L., Wu, Y., \& Zhang, B., et al. (2018). S1PR1 predicts patient survival and promotes chemotherapy drug resistance in gastric cancer cells through STAT3 constitutive activation. EBioMedicine . 37, 168-176. 10.1016/j.ebiom.2018.10.005

Song, S., Su, Z., Xu, H., Niu, M., Chen, X., \& Min, H., et al. (2017). Luteolin selectively kills STAT3 highly activated gastric cancer cells through enhancing the binding of STAT3 to SHP-1. Cell Death $\mathcal{G}$ Disease . 8(2), e2612. 10.1038/cddis.2017.38

Stitt, T.N., Drujan, D., Clarke, B.A., Panaro, F., Timofeyva, Y., \& Kline, W.O., et al. (2004). The IGF-1/PI3K/Akt Pathway Prevents Expression of Muscle Atrophy-Induced Ubiquitin Ligases by Inhibiting FOXO Transcription Factors. Molecular Cell . 14(3), 395-403. https://doi.org/10.1016/S10972765(04)00211-4

Tanaka, Y., Eda, H., Tanaka, T., Udagawa, T., Ishikawa, T., \& Horii, I., et al. (1990). Experimental cancer cachexia induced by transplantable colon 26 adenocarcinoma in mice. Cancer Res . 50(8), 2290-2295.

Trepel, J., Mollapour, M., Giaccone, G., \& Neckers, L. (2010). Targeting the dynamic HSP90 complex in cancer. Nat Rev Cancer . 10(8), 537-549. 10.1038/nrc2887

Ueki, K., Kondo, T., \& Kahn, C.R. (2004). Suppressor of cytokine signaling 1 (SOCS-1) and SOCS-3 cause insulin resistance through inhibition of tyrosine phosphorylation of insulin receptor substrate proteins by discrete mechanisms. Mol Cell Biol . 24(12), 5434-5446. 10.1128/MCB.24.12.5434-5446.2004

Whitesell, L., \& Lindquist, S.L. (2005). HSP90 and the chaperoning of cancer.Nature Reviews Cancer . 5(10), 761-772. 10.1038/nrc1716

Yu, C.L., Meyer, D.J., Campbell, G.S., Larner, A.C., Carter-Su, C., Schwartz, J., \& Jove, R. (1995). Enhanced DNA-binding activity of a Stat3-related protein in cells transformed by the Src oncoprotein.Science . 269(5220), 81-83. 10.1126/science.7541555

Yun, C.W., Kim, H.J., Lim, J.H., \& Lee, S.H. (2019). Heat Shock Proteins: Agents of Cancer Development and Therapeutic Targets in Anti-Cancer Therapy. Cells . 9(1). 10.3390/cells9010060

Zhang, L., Pan, J., Dong, Y., Tweardy, D.J., Dong, Y., Garibotto, G., \& Mitch, W.E. (2013). Stat3 activation links a C/EBPdelta to myostatin pathway to stimulate loss of muscle mass. Cell Metab . 18(3), 368-379. 10.1016/j.cmet.2013.07.012

Zhong, Z., Wen, Z., \& Darnell, J.J. (1994). Stat3: a STAT family member activated by tyrosine phosphorylation in response to epidermal growth factor and interleukin-6. Science . 264(5155), 95-98. 10.1126/science. 8140422 
Zimmers, T.A., Fishel, M.L., \& Bonetto, A. (2016). STAT3 in the systemic inflammation of cancer cachexia. Seminars in Cell \& Developmental Biology . 54, 28-41. 10.1016/j.semcdb.2016.02.009

\section{Figure Legends}

Figure 1. Enhanced HSP90-STAT3 interaction induced prolonged STAT3 activation in the skeletal muscle from cancer cachectic patients and C26 tumor-bearing cachectic mice.

The expression levels of Atrogin-1, MuRF-1, MHC, HSP90, Myostatin, and phosphorylated STAT3 in the skeletal muscle from cancer cachectic patients were detected. (a) the expressions of Atrogin-1 and MuRF1 were assessed by qRT-PCR (b) the expressions of Atrogin-1, MuRF-1, MHC, HSP90, Myostatin, and phosphorylated STAT3 were detected by Western blot assay. (c) Measurement of the HSP90-STAT3 interaction in cachectic muscle cancer cachectic patients by immunoprecipitation. (d, e) The expression levels of Atrogin-1, MuRF-1, MHC, HSP90, Myostatin, and phosphorylated STAT3 in the skeletal muscle from C26 tumor-bearing cachectic mice were detected by (d. qRT-PCR for atrogin-1 and MuRF-1 and e. Western blot for Atrogin-1, MuRF-1, MHC, HSP90, Myostatin, and phosphorylated STAT3). (f) The lysates of C26 or control mice muscle were immunoprecipitated with the STAT3 antibody, the HSP90 antibody, or control rabbit IgG. The immunopellets were detected by immunoblot analysis with the anti-HSP90 antibody and the anti-STAT3 antibody

Figure 2. Knocking down of HSP90 or STAT3 prevented C2C12 myotubes from C26 CMinduced atrophy.

(a) The expression levels of Atrogin-1, MuRF-1, MHC, HSP90, Myostatin, and phosphorylated STAT3 in the skeletal muscle from $\mathrm{C} 26 \mathrm{CM}$ treated $\mathrm{C} 2 \mathrm{C} 12$ myotubes were detected by western blot. (b) The lysates of C2C12 cells treated with C26 CM or DMEM (control) were immunoprecipitated with the STAT3 antibody, the HSP90 antibody, or control rabbit IgG. The immunopellets were detected by immunoblot analysis with the anti-HSP90 antibody and the anti-STAT3 antibody.(c) Representative hematoxylin and eosin (HE)stained C2C12 treated with C26 CM and transfected with the HSP90 siRNA or the STAT3 siRNA. (d) The histogram represents the average myotube diameter $(\mu \mathrm{m})$ (d, left part) . qRT-PCR analysis of atrogin-1 and MuRF-1 in the gastrocnemius muscles of mice (d, right part) .(e) Atrophy markers in $\mathrm{C} 2 \mathrm{C} 12$ were tested by WB analysis when the expression of HSP90 or STAT3 was knocked down by siRNA. Data represent the mean \pm standard deviation (SD). ${ }^{*} \mathrm{p}<0.05,{ }^{* *} \mathrm{p}<0.01$ denotes compared to control; $\# \mathrm{p}<0.05, \# \# \mathrm{p}<0.01$ denotes compared to $\mathrm{C} 26 \mathrm{CM}$ treated myotubes.

Figure 3. Administration of HSP90 inhibitor 17DMAG prevented C26 CM-induced myotube atrophy and disrupted the binding between HSP90 or JAK2 with STAT3.

(a) $\mathrm{C} 2 \mathrm{C} 12$ myotubes were treated with C26 CM, C26 CM plus HSP90 inhibitor 17DMAG (0.1 umol), or normal media for $24 \mathrm{~h}$. $\mathrm{H} \& \mathrm{E}$ staining shows the morphological changes in $\mathrm{C} 2 \mathrm{C} 12$ myotubes. Scale bar: 50 $\mu \mathrm{m}$. (b) The histogram represents the average myotube diameter $(\mu \mathrm{m})$ and myotube area $\left(\mu \mathrm{m}^{2}\right)$ in control, CM-treated, and 17DMAG plus CM-treated $\mathrm{C} 2 \mathrm{C} 12$ myotubes. The number of myotubes was quantified using ImageJ. qRT-PCR analysis of atrogin-1 and MuRF-1 in the gastrocnemius muscles of mice (b, right part). (c) $\mathrm{C} 2 \mathrm{C} 12$ cells were immunostained with the MHC (MF20) antibody. MHC, green, DAPI, blue. The expression and subcellular localization of myostatin and pY-STAT3 in C2C12 myotubes were detected by immunofluorescence staining. Myostatin and pY-STAT3, green, PI, blue. Scale bar: $30 \mu \mathrm{m}$. (d) The expression of MHC, HSP90, pY-STAT3, atrogin-1, and MuRF-1 in control, CM-treated, and 17DMAG plus CM-treated $\mathrm{C} 2 \mathrm{C} 12$ myotubes was analyzed by western blot, GAPDH served as a loading control. (e) The lysates of $\mathrm{C} 2 \mathrm{C} 12$ cells treated with C26 CM or DMEM (control) were immunoprecipitated with the STAT3 antibody, the HSP90 antibody, or control rabbit IgG. The immunopellets were detected by immunoblot analysis with the anti-HSP90 antibody and the anti-STAT3 antibody. Data represent the mean \pm standard deviation (SD). ${ }^{*} \mathrm{p}<0.05,{ }^{* *} \mathrm{p}<0.01,{ }^{* * *} \mathrm{p}<0.001,{ }^{*} * * * \mathrm{p}<0.0001$ denotes compared to control; $\# \mathrm{p}<0.05$, \#\# $<0.01$, \#\#\# $<0.001$ denotes compared to $\mathrm{C} 26 \mathrm{CM}$ treated myotubes.

Figure 4. The protective effects of 17DMAG in cachectic muscle wasting in C26 tumor-bearing 
mice.

(a) Left part : Bodyweight changes in normal control mice, 17DMAG or PU-H71-treated C26 tumorbearing mice; veh1(17DMAG solvent) or veh2(PU-H71 solvent) treated C26 tumor-bearing mice. Day 0: tumor implantation. Day 7: 17DMAG injection began. Day 15: Terminal sacrifice day. Right part : Another experiment for survival rates of C26-tumor-bearing mice that received the treatment of 17DMAG or PU-H71 and their vehicle control. (b) Representative images of the hindlimbs of normal (left), C26 tumor-bearing (middle), and 17DMAG-treated C26 tumor-bearing (right) mice. (c) Grip strength was measured on day 14. (d) Tumor-free body mass, lean mass weight, and the tibialis anterior (TA), gastrocnemius (Gastroc), soleus, and extensor digitorum longus (EDL) muscles were weighed and compared after dissection with an electronic scale; Data represent the mean \pm standard deviation (SD). ${ }^{*} \mathrm{p}<0.05,{ }^{* *} \mathrm{p}<0.01,{ }^{* * *} \mathrm{p}<0.001,{ }^{* * * *} \mathrm{p}<0.0001$ denotes 17DMAG vs. C26; \#p $<0.05$, \#\#p $<0.01$, \#\#\#p $<0.001$ denotes $\mathrm{C} 26$ vs. control.

Figure 5. Protective Effects of 17DMAG on fiber cross-sectional area, STAT3 activation, and catabolic pathway suppression.

(a) Representative hematoxylin and eosin (H\&E) staining showing the morphological changes in the gastrocnemius. Scale bar: $100 \mu \mathrm{m}$. (b) Fiber size distribution. (c) Mean fiber cross-sectional area is shown by the $\chi^{2}$ analysis $($ for $\mathrm{CSA}) ;{ }^{*}$ denotes a significant difference $(\mathrm{P}<0.05)$ compared with normal control; \# denotes a significant difference $(\mathrm{P}<0.05)$ compared with $\mathrm{C} 26$ mice. $($ d) $17 \mathrm{DMAG}$ treatment altered the expressions of myosin heavy chain (MHC), MuRF-1, atrogin-1, and pY-STAT3 in cachectic skeletal muscles from C26 tumor-bearing mice, which were detected by western blot analysis. (e) Gastrocnemius MHC expression was evaluated by immunofluorescence staining. MHC, green, DAPI, blue. Scale bar: $100 \mu \mathrm{m}$. (f) Total RNA was extracted from the gastrocnemius from control, 17DMAG-treated, and C26tumor-bearing mice, and the expression levels of MuRF-1 and atrogin-1 in the gastrocnemius were measured by quantitative RT-PCR. Data represent the mean $\pm \mathrm{SD} .{ }^{* * *} \mathrm{p}<0.001$, compared with normal control; $\# \mathrm{p}<0.05$, compared with $\mathrm{C} 26$ mice.

Figure 6. STAT3 regulated FOXO1 expression is responsible for C26 CM-induced myotube atrophy.

(a) Western blot analysis of FOXO1 expression in skeletal muscle from $\mathrm{C} 26$ cachectic mice and $\mathrm{C} 2 \mathrm{C} 12$ myotubes with or without 17DMAG treatment. (b) Representative Western blot assay of the FOXO1 expression after knocking down STAT3 by siRNA. (c)Representative immunofluorescence microscopy images showing the subcellular localization of FOXO1 in C2C12. (d) Schematic structure of the FoxO1 promoter region (NCBI Reference Sequence: NC_000069.5 for FoxO1). The transcription start site (TSS) is denoted by a black arrow. (e-f) Chromatin immunoprecipitation (CHIP) analysis was performed with $\mathrm{C} 2 \mathrm{C} 12$, and STAT3 binding to the FoxO1 promoter region was analyzed. Cell lysates were immunoprecipitated with antiSTAT3 Ab or control IgG. Immunoprecipitated and input DNA was analyzed by semi-quantitative PCR (e) or qPCR (f)using primers corresponding to FoxO1 promoter sites. (g)Representative Western blot assay of FOXO1, p-FOXO1, and atrophy genes expression in C2C12 myotube co-transfected with FOXO1 siRNA and STAT3-C recombinant plasmid. (h) Representative Western blot of FOXO1, p-FOXO1, and atrophy genes expression in $\mathrm{C} 2 \mathrm{C} 12$ myotube transfected with or without mutated forms (constitutive activated) of STAT3 overexpression plasmid (Stat3-C) and treated with or without 17DMAG. 
Figure 1

(a)

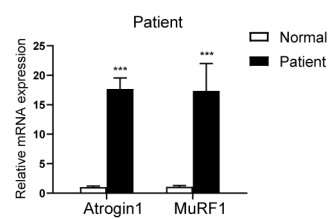

(b)

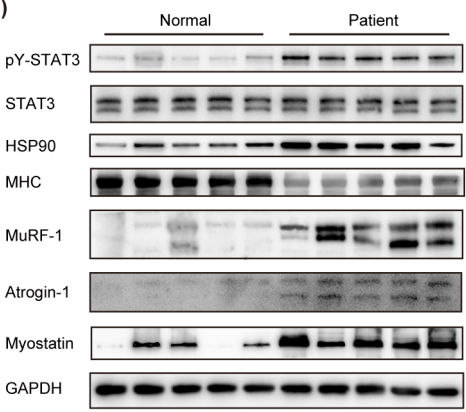

(c)

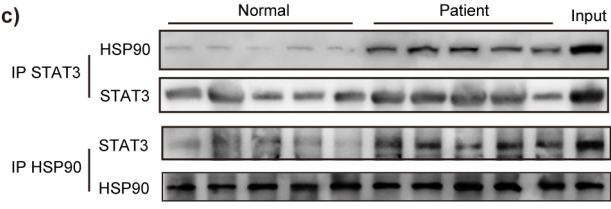

(d)

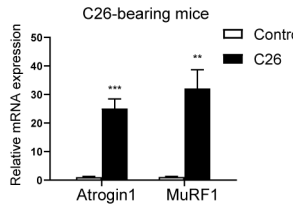

(e)

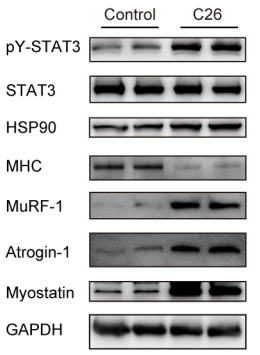

(f)

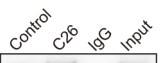

IP: STAT3 $\left.\right|_{\text {STAT3 }} ^{\text {HSP90 }}$

IP: HSP90 $\left.\right|_{\text {HSP90 }} ^{\text {STAT3 }}=-$

GAPDH $=0$

Figure 2

(a)

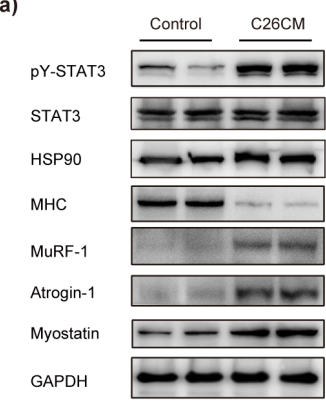

(b)

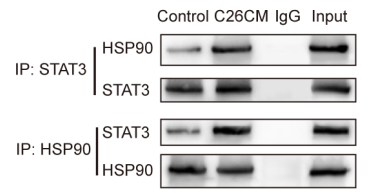

(c)

HE

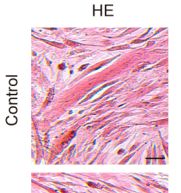

स- $=$.
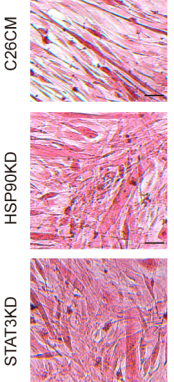

(d)
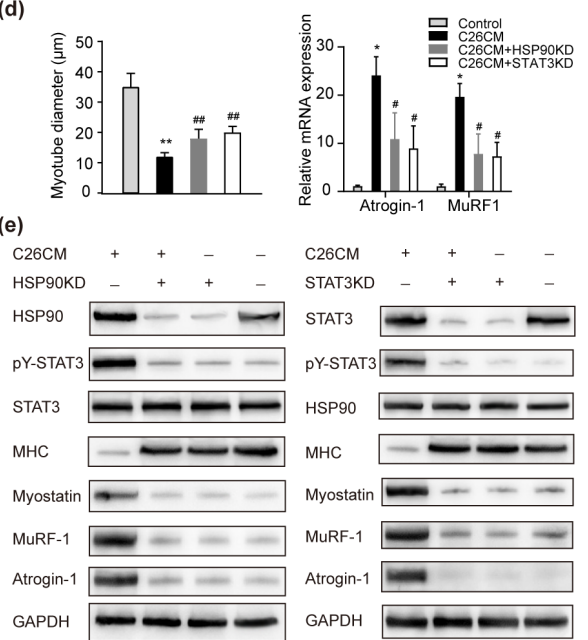

Figure 3

(a)

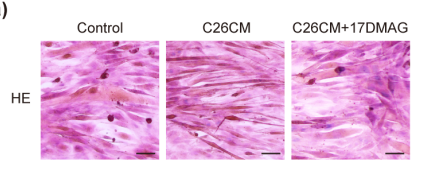

(c)

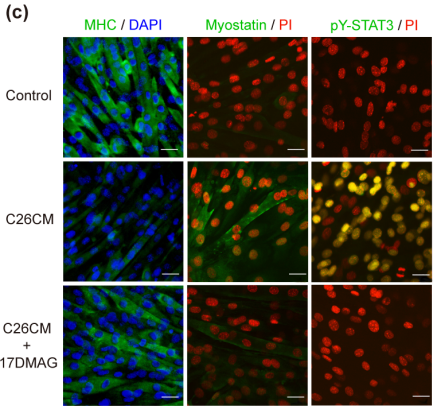

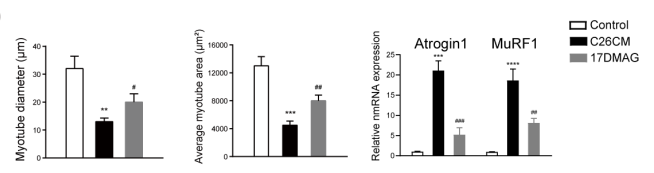

(d)

(e)

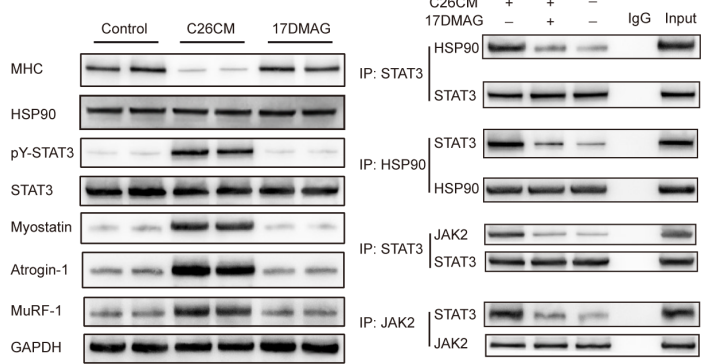


Figure 4
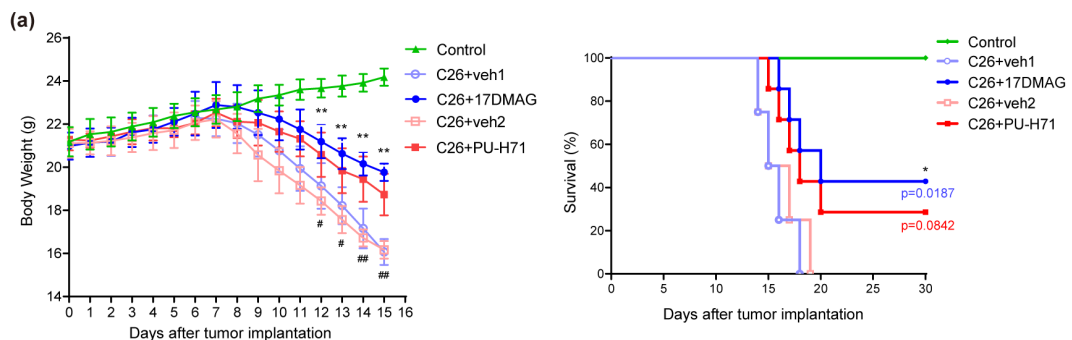

(b)

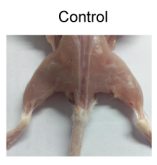

(d) Tumor-free body weight
C26+veh1
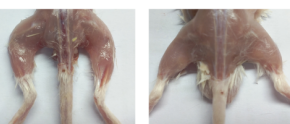

Lean mass

(c)
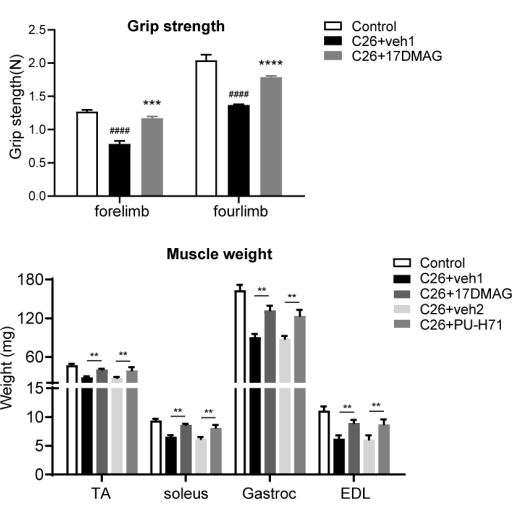

Figure 5

(a)

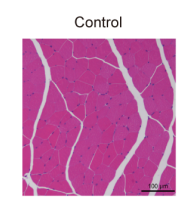

(b)

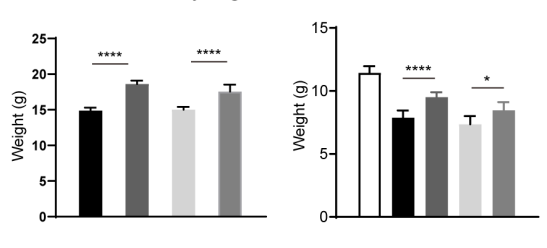

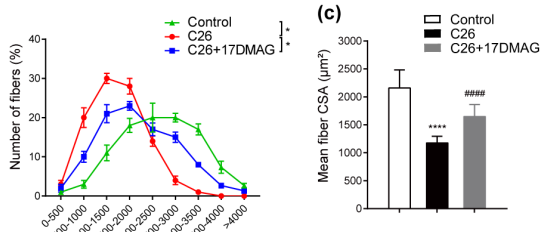

(e)

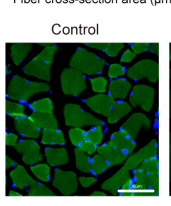

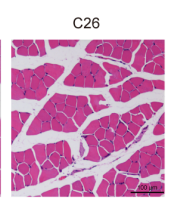

$\mathrm{C} 26$

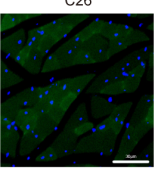

C26+17DMAG

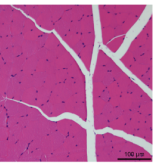

C26+17DMAG

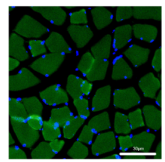

(d)

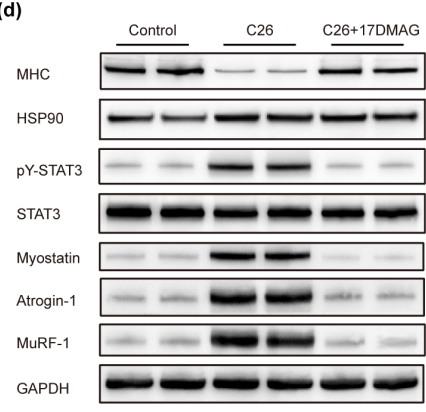

(f)

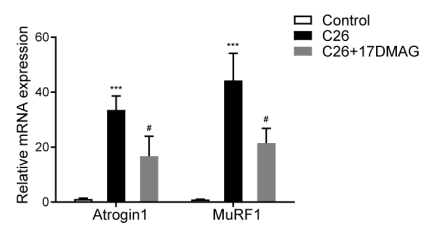




\section{Figure 6}

(a)

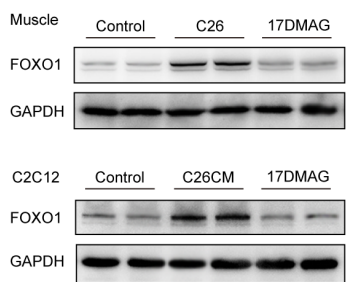

(b)
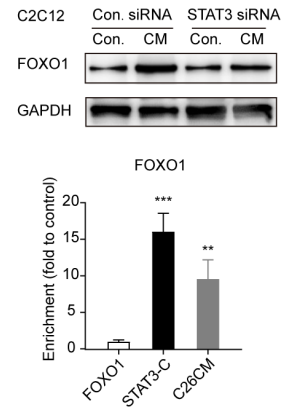

(c)

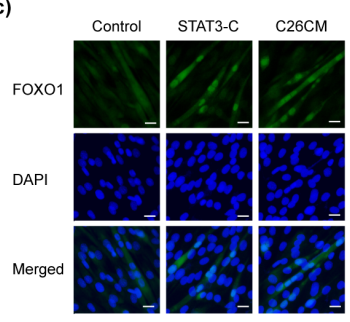

(g)

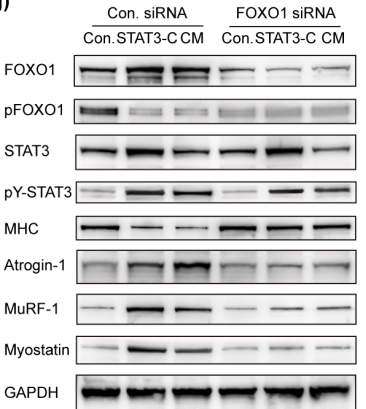

(d)

(e)

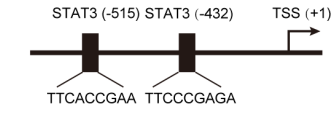

FOXO1

Control Ab Control STAT3-C C26CM

IP

Input DNA $=0$

(h)

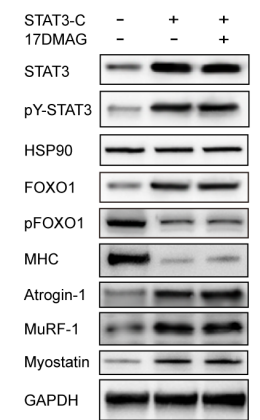

